

\section{REVISTA \\ TEORÍA Y PRÁCTICA \\ DE LA \\ ARQUEOLOGÍA HISTÓRICA LATINOAMERICANA}

ISSN: 2250-866X (impreso) | ISSN: 2591-2801 (en línea)

AÑo VI, Volumen 6, Verano DE 2017

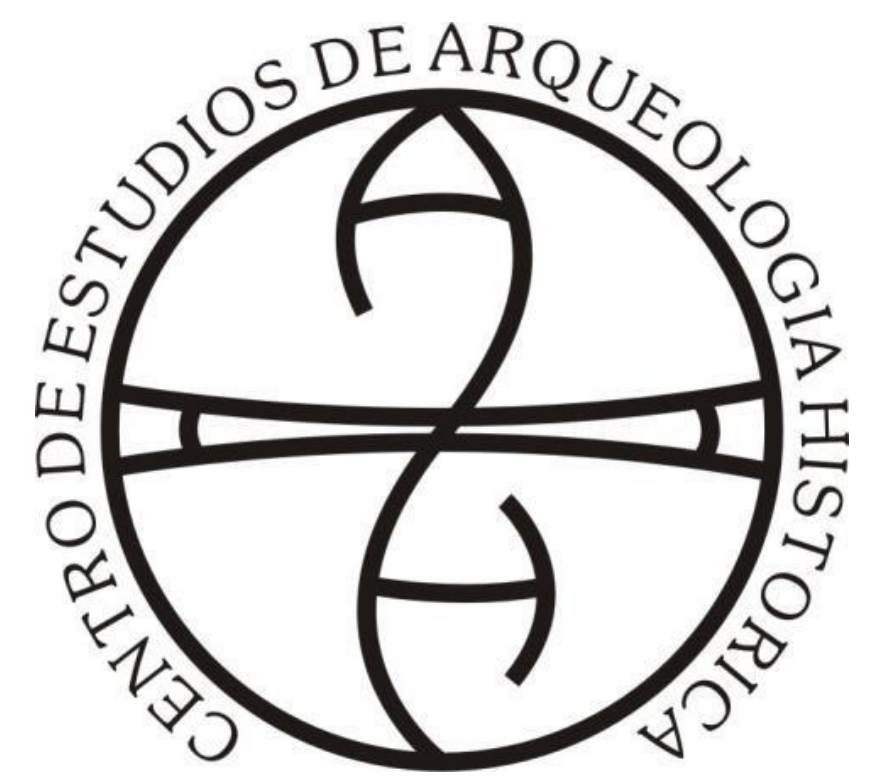

Centro de Estudios de Arqueología Histórica

FACULTAD DE HUMANIDADES Y ARTES | UNIVERSIDAD NACIONAL DE ROSARIO 

PARTICIPA EN LA RED DE ESTUDIOS INTEGRADOS SOBRE LOS PAISAJES SUDAMERICANOS

(Universidad Nacional de Rosario, Universidad Nacional de Río Cuarto,

Universidad Nacional de San Juan, Universidad de la República, Universidad Nacional de Trujillo)

AUTORIDADES DE LA UNIVERSIDAD NACIONAL DE ROSARIO

Rector: Dr. Arq. Héctor Floriani

Vicerrector: Lic. Fabián Bicciré

Secretario general: Lic. Mariano Balla

Secretario académico: Dr. Carlos A. Hernández

\author{
AUTORIDADES DE LA FACULTAD DE HUMANIDADES Y ARTES \\ Decano: Prof. José Goity \\ Vicedecano: Prof. Marta Varela \\ Secretaria Académica: Dra. Liliana Pérez
}

AUTORIDADES DEL CENTRO DE ARQUEOLOGÍA HISTÓRICA

Directora: Dra. Ana María Rocchietti

Secretaria: Prof. Nélida De Grandis

Prosecretaria: Lic. Marianela Biscaldi

Revista del Centro de Estudios de Arqueología Histórica

Teoría y Práctica de la Arqueología Histórica Latinoamericana Actas del Sexto Simposio de Arqueología Histórica Latinoamericana

Presidente del Simposio: Licenciada Mónica Valentini
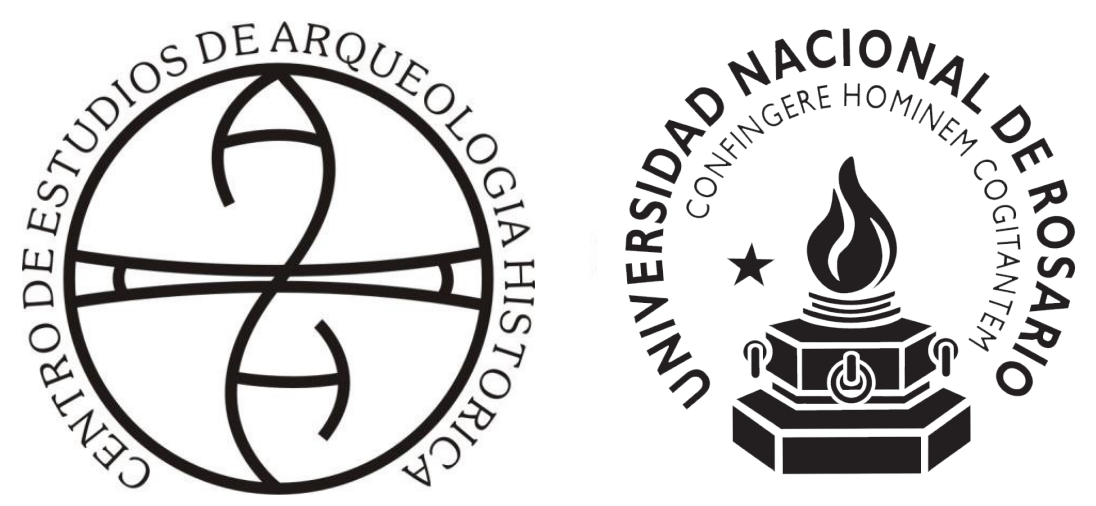


\section{Directoras Editoras}

Ana María Rocchietti y Nélida De Grandis

\section{Secretaria}

Irene Dosztal

\section{Comité Editor}

Ana María Rocchietti, Mónica P. Valentini

Nélida De Grandis, Marianela Biscaldi

Irene Dosztal, María Victoria Roca,

Fátima Solomita Banfi

\section{Comité Científico del V Simposio}

Dra. Nidia Areces

Dr. Raúl Bolmaro

Dr. Luis María Calvo

Lic. Juan Castañeda Murga

Lic. Carlos Ceruti

Dr. Juan Bautista Leoni

Dra. Eugenia Néspolo

Lic. Ruth Adela Poujade

Ing. Adrián Pifferetti

Dr. Mariano Ramos

Dr. Daniel Schávelzon

Dra. Marcela Tamagnini

Dra. Alicia Tapia

\section{Comité Científico}

Dr. Raúl Bolmaro (Universidad Nacional de Rosario)

Dr. Luis María Calvo (Museo Etnográfico de Santa Fe y Parque Arqueológico de Santa Fe La Vieja)

Lic. Carlos Ceruti (CONICET)

Dra. Dora de Grinberg (Facultad de Ingeniería, UNAM)

Dra. Eugenia Néspolo (Universidad Nacional de Luján)

Ing. Tulio Palacios (Comisión Nacional de Energía Atómica)

Lic. Ruth Poujade (Programa Misiones

Jesuíticas - Provincia de Misiones)

Dr. Mariano Ramos (Universidad Nacional de Luján)

Dra. Ana María Rocchietti (Universidad

Nacional de Rosario)
Dr. Daniel Schávelzon (Centro de

Arqueología Urbana - Universidad de

Buenos Aires)

Dra. Marcela Tamagnini (Universidad

Nacional de Río Cuarto)

Dra. Alicia Tapia (Universidad de Buenos

Aires)

Dr. Arno Álvarez Kern (Centro Nacional de

Pesquisas - Brasil)

Dra. Noemí Walsöe de Reca (CONICET)

Lic. Mónica Valentini (Universidad

Nacional de Rosario)

\section{Evaluaron este Volumen}

Horacio Chiavazza, Mónica Valentini,

Nélida De Grandis, Mariano Ramos, Juan

Bautista Leoni, Ana María Rocchietti, Javier García Cano, Flavio Ribero

\section{Diseño y diagramación}

Eugenia Reboiro

(eugenia.reboiro@gmail.com)

\section{Curaduría}

Irene Dosztal y Ana María Rocchietti

Foto de tapa: botellas de vidrio del texto de Orsini y Padula.

\section{Propietario responsable:}

Universidad Nacional de Rosario

Facultad de Humanidades y Artes,

Centro de Estudios de Arqueología

Histórica.

Entre Ríos 758. Rosario, Provincia de Santa

Fe (2000). Argentina.

Telf.: +54 (0341) 4802670

E-mail: ceahunr@gmail.com

Decreto Ley 6422/57 de Publicaciones Periódicas 


\section{Índice}

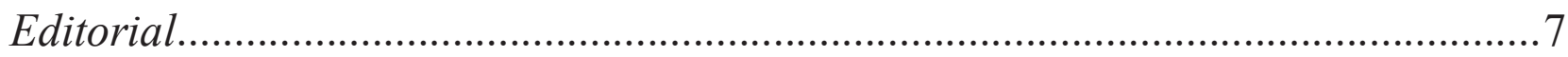

Experimentación, transferencia tecnológica y conservadurismo: notas sobre la introducción del aforro de cobre en los barcos de madera del siglo XVIII......

Nicolás C. Ciarlo

Arqueología social latinoamericana y los desafios de la arqueología urbana.

Ana María Rocchietti

"No todo fue hispánico en la casa de los Larreta". Intervención arqueológica en el jardín del Museo Larreta.

Ricardo Orsini y Horacio Padula

La construcción con ladrillo asentado en barro. El caso de la ciudad de

Rosario

Adrián A. Pifferetti

“Mientras las gárgolas nos miran”. Un sitio arqueológico urbano ¿y religioso?

en la ciudad de Luján. Provincia de Buenos Aires.

Mariano Ramos, Alejandra Raies y Matías Warr

Asentamientos europeos en la cuenca del Río de la Plata: primeras

aproximaciones a Corpus Christi.

Fernando Balducci, Julieta Sartori, Paula Galligani, Fernando Roggero, Macarena Riberi y Abril Castagnola

"De lugares y objetos". La visibilización del pasado afro en el norte de Argentina. Caso de estudio: la ciudad colonial de San Miguel de Tucumán.... 79

Luciana Chávez

Teledetección espacial aplicada a la búsqueda de restos de la Estancia San Miguel del Carcarañá.

Fabián Bognanni, Mónica P. Valentini, Mariano Darigo, Matías Warr y María Lara Moschetoni 
Rescate arqueológico. Hotel Pensión Europea. Posadas.

Misiones. Argentina.

Marianela Biscaldi y Lorena Salvatelli

El Fuerte Independencia revive tres siglos después en medio de un debate sobre sus túneles

Julio Fabián Merlo y María del Carmen Langiano

Estudio de muestras metálicas de vado de Las tres cascadas, Río Cuarto, Córdoba

Adrián A. Pifferetti

Clasificación de botones Prosser y su potencial como indicador

cronológico. Arqueología urbana de Rosario (sitio La Basurita)

María Fernanda Bruzzoni y Sandra Escudero

Una casa del siglo XX y un fuerte del siglo XVI. Arqueología

histórica en Cobos.

Jorge Esteban Cabral Ortiz y Rosana Jimena Flores

Un fechado intrigante

Mario Silveira y Mónica Patricia Valentini

Criterios adoptados para la conservación de los restos de estructura naval del Pecio Zencity de Puerto Madero (Buenos Aires, Argentina): ¿¿RE-ENTERRAR PARA CONSERVAR?!.

Eva Tavella 


\title{
Clasificación de botones Prosser y SU POTENCIAL COMO INDICADOR CRONOLÓGICO. ARQUEOLOGÍA URBANA De Rosario (SITIO La BASURITA)
}

\author{
María Fernanda Bruzzoni y Sandra Escudero *
}

\begin{abstract}
Resumen
Los botones Prosser son un tipo particular de botones de cerámica de producción industrial cuya expansión comercial se desarrolló entre1840 y 1950/1960 aproximadamente, cuando fueron desplazados por el plástico. Pese a su frecuente presencia en sitios arqueológicos del siglo XIX, en la literatura arqueológica sólo se los menciona a título cuantitativo o descriptivo, sin otorgarles mayor relevancia informativa.

El sitio arqueológico la Basurita (LB), primer vaciadero municipal de Rosario durante la segunda mitad del siglo diecinueve, presenta una gran cantidad y variabilidad de este tipo de botón que ha posibilitado su análisis.

Con base en la tesis de grado en Antropología de una de las autoras, en este trabajo se presentan las tipologías de botones Prosser hallados en el sitio LB, y se introduce su potencial en cuanto a indicador cronológico de sitios arqueológicos del siglo XIX gracias a su acotada y confiable cronología de inicio y expansión comercial.
\end{abstract}

Palabras clave: botones Prosser-indicador cronológico - siglo 19- Rosario

\begin{abstract}
Prosser buttons are a particular type of industrial ceramic buttons with an commercial success around 1840-1950/1960, then plastic left them out of the market. Despite their ubiquity in19th century archaeological sites, in the literature they are mentioned just in quantitative or descriptive ways, without an informative consideration.

A large quantity and variability of Prosser buttons were recovered from La Basurita(LB) archaeological site, the first municipal dum of Rosario city throughout the second half of the nineteenth century, presents a great that has made its analysis possible.

From the previous analysis made for one of the authors in the frame of her degree thesis in Anthropology, in this work are presented the typologies of Prosser buttons from the LB site, and ii is introduced its potential as a chronological indicator of archaeological sites of the nineteenth century thanks both its limited and reliable starting chronology and commercial expansion.
\end{abstract}

Keywords: Prosser buttons - chronological indicator - 19th century - Rosario city

\footnotetext{
* Departamento de Arqueología. ESM, Municipalidad de Rosario. fernandabruzzoni@gmail.com; cescude0@rosario. gov.ar
} 


\section{Sitio La Basurita}

El sitio arqueológico La Basurita (LB) se encuentra ubicado entre las calles Pasco, Ituzaingó, Cerrito, Berutti y la barranca de Av. Belgrano de la ciudad de Rosario, Santa Fe, Argentina. Allí funcionó el primer vaciadero municipal entre los años 1870 y 1890 aproximadamente. También es conocido como "La Pólvora, Basura Vieja, Antiguo Vaciadero o Barrio La Quema" (Volpe, 1994, p.83)

La historia ocupacional del sitio estuvo fuertemente vinculada al ejército, ya que en la zona hacia el año 1859, se emplazaban las Baterías de la Confederación, y posteriormente, entre 1867 y 1869, fue utilizado en la Guerra del Paraguay.

Ya finalizada la guerra, el polvorín estalló dejando un profundo pozo que llevó a la habilitación del lugar como Vaciadero Municipal desde 1873 hasta 1890 aproximadamente (Volpe, 1994).

En un primer momento entre los años 1902 y 1930, la morfología de este sitio se caracterizaba por un montículo artificial aplanado, cortado y expandido durante la construcción del puerto y la avenida Belgrano. Entre 1970 y 1980 se realizaron tareas de nivelación del terreno por parte de la Universidad Nacional de Rosario presentando hoy un aspecto que se confunde con la barranca (Volpe, 1994).

El Departamento de Arqueología de la Escuela Superior de Museología de Rosario (ESM), inició y desarrolló las investigaciones del sitio a través de trabajos de prospección y excavación realizados bajo la dirección de Soccorso Volpe entre los años 1988 y 1993. Se ejecutaron una serie de trincheras y cuadrículas y varios pozos de sondeo, además de recolecciones superficiales asistemáticas que no quedaron registradas. Según Volpe (1992), el suelo del sitio estaba compuesto por tierra suelta con presencia de carbón, capas de óxido y diferentes sales que pertenecían a elementos químicamente degradados.

Durante las actividades se detectaron estructuras enterradas y semienterradas que se asumieron como pertenecientes a diversas actividades a través del tiempo ligadas al comercio en general, como fábricas, oficinas, depósitos, barracas, etc. (Volpe, 1988 y 1994).

Los materiales recuperados se encuentran actualmente en el Departamento de Arqueología de la Escuela Superior de Museología y presentan distintos grados de conservación y preservación. Comprenden tanto restos cerámicos (loza, gres, porcelana), vítreos de distintos tipos, elementos de metal, restos óseos de fauna, semillas, cuero y papel, entre otros. Dentro de los materiales cerámicos se recuperó una cantidad importante de botones tipo Prosser que constituyen el objeto de esta comunicación

Volpe (1992) agrupó todos los elementos en rubros tales como zapatería, ferretería, electricidad, mercería, artículos de tocador, droguería, etc. En cuanto a los diferentes tipos de botones recuperados fueron inicialmente adheridos con pegamento a hojas de papel milimetrado. Durante el proceso de normalización de colecciones del Departamento de Arqueología iniciado en 2004 fueron removidos, oportunamente limpiados, separados por material, re-marcados y colocados en bolsas individuales ubicadas en cajas de cartón para su orden y conservación. Ningún tipo de botón, incluido los Prosser, había sido objeto de estudio de ningún investigador hasta la fecha, sólo en ocasiones se los refirió nominalmente.

\section{Botones Prosser}

Pese a su ubicuidad en sitios arqueológicos de la segunda mitad del siglo XIX, los botones Prosser han atraído poca atención por parte de los investigadores. El artículo de Sprague (2002) continúa siendo la referencia principal y asimismo sigue siendo válida su apreciación de utilidad de los coleccionistas de botones por su sistema clasificatorio. 
Los botones Prosser son un tipo de botón de cerámica fabricados en Inglaterra hacia 1840 por los hermanos Richard y Thomas Prosser. El proceso industrial llamado dust (polvo) o Prosser consistía en una preparación de arcilla fina mezclada con cuarzo o cerámica molida algo húmeda que se prensaba en moldes de hierro fundido y se cocinaba en hornos mufla a temperatura lo suficientemente alta como para transformar la arcilla en botones de cerámica logrando el nivel de la porcelana. Retirados de la primera cocción, los botones se podían pintar de colores, realizar impresiones o transferencias como los tipos calicó y gingham. Y por último, pasaban al segundo horno para el acabado final (Sprague, 2002). La característica que diferencia este tipo de botón con el resto de los botones de cerámica y/o con los de vidrio es su superficie de grava o "cáscara de naranja" en la parte posterior del mismo debido a las marcas propias del golpe de la prensa sobre la matriz (Sprague, 2002).

En el año 1843, Jean-Félix Bapterosses, un industrial e inventor francés, creó una prensa capaz de producir por golpeo 500 piezas de botones a la vez. A su vez incorporó matrices intercambiables que le permitió fabricar botones no solo con agujeros sino también con ganchos, y le incorporó leche (caseína) a la masa por lo que se volvió más plástica que la masa tradicional inglesa. Gracias a la mejora en la maquinaria, la modificación en la masa y la mano de obra más barata, la fabricación en Francia terminó siendo la más económica y arrasó con el negocio en Inglaterra (Sprague, 2002; Ebelmen y Salvetat, 1855). Su bajo costo permitió que estuviera al alcance de todos y rápidamente se hicieron más populares. El uso de técnicas como el stencil (estarcido) permitía la producción de botones de apariencia tal que fueron una alternativa a los botones de tela (Gorski, 2009).

Según Sprague (2002) no hay certeza acerca de hasta qué fecha se fabricaron botones de tipo Prosser, pero su declive fue notorio tras la aparición del plástico hacia 1950-1960 el cual tuvo un impacto definitivo en su producción.

\section{Botones Prosser presentes en el Sitio LB}

Los botones Prosser se pueden dividir en tres grandes categorías de acuerdo a su método de fijación a la prenda de vestir: Categoría SewThrough-Agujeros-, Categoría Shank-Ganchos- con un dispositivo en la parte posterior tal como una placa y bucle de metal (Gorski, 2009, National Button Society 2011-2012 - en adelante NBS 2011-2012-) y Categoría Complex Construction -Construcción Compleja- que abarca a los botones fabricados en piezas separdas y encajadas, de construcción sólida o hueca con un solo agujero en la parte superior del botón y dos o tres agujeros en la parte posterior (NBS, 2011-2012).

El registro arqueológico del sitio La Basurita consta aproximadamente de once mil quinientos elementos. Entre ellos se recuperaron 656 botones de los cuales 481 son de tipo Prosser y el resto de otras materialidades: nácar $(n=80)$, madera o hueso $(n=43)$, vidrio $(n=12)$, plástico $(n=8)$, metal $(\mathrm{n}=1)$, madera y nácar $(\mathrm{n}=1)$ y porcelana no $\operatorname{Prosser}(n=30)$.

De los 481 botones Prosser, se analizaron 452 correspondientes a las categorías de fijación SewThrough -Agujeros- (Gorski, 2009) y Complex Construction - Construcción Compleja- (NBS 2011-2012). Los restantes 29 pertenecen a la categoría Shank (Gancho) que serán considerados en trabajos posteriores ya que no son puramente de cerámica sino que en su proceso de fabricación incorporan otra materialidad -metal- en su estructura. Y dentro de la muestra analizada, 422 fueron determinados y 30 indeterminados dada su escasa o nula identificación como botones

A continuación, y siguiendo la clasificación del extracto de la Guía para coleccionistas de botones tipo China (Lamm et al., 1970), se desarrollarán los diferentes tipos de botones que se encuentran presentes en el sitio LB.

Dentro de la categoría de fijación“Agujeros" se encuentran botones de cuatro agujeros (n=212), botones de dos agujeros $(n=62)$ y botones de tres agujeros $(n=6)$ con sus respectivos subtipos. En la Categoría "Construcción compleja" se halló sólo el tipo silbato $(n=142)$. Estos botones no están 
denominados en Lamm (et.al., 1970) sino que solo están referenciados con el nombre de figura y un número. A los fines de este trabajo y de próximas investigaciones, se les dio un nombre siguiendo la lógica identificadora en las designaciones en uso para los otros botones Prosser

La cantidad y distribución de tipos y subtipos de la categoría Agujeros (dos, tres y cuatro agujeros) se especifican en las Tablas 1, 2 y 3. En cuanto a los botones de la Categoría Construcción Compleja se hallaron sólo los del tipo Whistle (Silbato) con los distintos subtipos cuya distribución se detalla en la Tabla 4.

Tabla 1.

Distribución de tipos en la Categoría Cuatro Agujeros

\begin{tabular}{llcc}
\hline & TIPO & CANTIDAD & PORCENTAJE \\
\hline \multirow{4}{*}{ CUATRO } & Platillo (Saucer) & 7 & 3,30 \\
& Borde Tachuela (HobnailRim) & 8 & 3,77 \\
AGUJEROS & BordeLíneasRadiales (Radianting Line Rim) & 4 & 1,89 \\
& Borde Curvo (RolledRim) & 9 & 4,25 \\
& BordeBiselado (Smooth Beveled Rim) & 184 & 86,79 \\
& TOTAL & 212 & $100 \%$ \\
\hline
\end{tabular}

Tabla 2.

Distribución de tipos en la Categoría Dos Agujeros

\begin{tabular}{llll}
\hline & \multicolumn{1}{c}{ TIPO } & CANTIDAD & PORCENTAJE \\
\hline \multirow{2}{*}{ DOS AGUJEROS } & Hueco (HollowEye) & 28 & 45,16 \\
& Hueco Profundo (Deepwell) & 4 & 6,45 \\
& Hueco Ovalado (Oval Eyes) & 25 & 40,32 \\
& Decorado (PatternEyes) & 5 & 8,06 \\
& TOTAL & 62 & $100 \%$ \\
\hline
\end{tabular}

Tabla 3.

Distribución de tipos en la Categoría Tres Agujeros

\begin{tabular}{llll}
\hline & TIPO & CANTIDAD & PORCENTAJE \\
\hline \multirow{2}{*}{ TRES AGUJEROS } & Plato (Dish) & 5 & 83,33 \\
& BordeLíneasRadiales (Radiating Line Rims) & 1 & 16,67 \\
& TOTAL & 6 & $100 \%$ \\
\hline
\end{tabular}


Tabla 4.

Categoría Construcción Compleja: Subtipos de botones Whistle

\begin{tabular}{llll}
\hline \multicolumn{4}{c}{ CATEGORIA CONSTRUCCION COMPLEJA } \\
\hline TIPO & SUBTIPO & CANTIDAD & PORCENTAJE \\
& Domo Bajo (Fig.1) & 7 & 4,93 \\
& Domo con Borde (Fig.2) & 7 & 4,93 \\
& Centro Hueco (Fig.3) & 10 & 7,04 \\
& Centro Hueco Decorado & 2 & 1,41 \\
& (Fig.3 Ilustrado) & & 26,06 \\
& Domo (Fig.4) & 37 & 3,52 \\
& Líneas Radiales (Fig.6) & 5 & 2,11 \\
& Bola de Fuego (Fig.7) & 3 & 1,41 \\
& Cono (Fig.8) & 2 & 5,63 \\
& Cono con Borde (Fig.9) & 8 & 0,70 \\
& Esfera (Fig.10) & 1 & 33,10 \\
& Esfera con borde (Fig.12) & 47 & 2,11 \\
& Ovni (Fig.14) & 3 & 3,52 \\
& Borde Elevado (Fig.15) & 5 & 2,11 \\
& Esfera Radial (Fig.20) & 3 & 0,70 \\
& Píldora (Fig. S/Catalogar) & 1 & 0,70 \\
& Tachuela con Anillos (Fig. S/Catalogar) & 1 & $100 \%$ \\
\hline
\end{tabular}

\section{Botones Prosser y su potencial como indicadores cronológicos}

Los botones en general, son uno de los elementos más comunes de hallar en sitios arqueológicos históricos. Por ejemplo botones con nácar en el sitio Iraolaen las cercanías de la localidad de La Plata, de Giovanettiy colaboradores (2001), el trabajo de Schávelzon (1991) sobre los materiales hallados en distintas excavaciones de Buenos Aires donde se recuperaron botones de vidrio, metal, asta, resina, hueso y nácar, el caso del sitio Vuelta Obligado de Lanza y colaboradores (2015) con un botón militar y un botón de nácar, el trabajo de García (2006) sobre el sitio La Merced en Mendoza capital con botones de vidrio. Y así sigue una lista de investigaciones en la Argentina donde solo en algunos casos se hace una aproximación de análisis de los mismos y en otros solo se los nombra de manera descriptiva, dejando "abierta la invitación para que este tema se siga desarrollando en profundidad por otros autores" (Schávelzon, 1991). Con la llegada de la Revolución Industrial y sus cambios sociales, los botones se vuelven una constante en el registro arqueológico como es el caso en particular de los botones Prosser encontrados frecuentemente a través de la segunda mitad del siglo XIX. Sin embargo, y como ya señaló Rivers (1999), en la mayoría de las investigaciones sólo se los limita a cuantificaciones y descripciones someras poniendo mayor interés en otras materialidades tales como las cuentas de cerámica, artefactos arquitectónicos, fragmentos de vidrio, restos arqueofaunísticos, etc. En el ámbito pampeano, los casos mencionados arriba ejemplifican esta tendencia: los botones son reducidos a cuantificaciones y descripciones someras.

Como cualquier otro material arqueológico, los botones pueden ser analizados para la obtención de datos y la construcción de información sobre conductas sociales: "El estudio de accesorios de 
vestir, objetos que, aunque están diseñados para ser funcional, también reflejan gustos personales, nos acerca a las necesidades y preferencias de las personas que poblaron el pasado" (Cox, 1996, p.58).

Como señaló Sprague (2002), gracias a su acotada y confiable cronología de inicio y expansión mercantil los botones Prosser ofrecen un excelente terminus post quem 1840. Como se dijo más arriba, si bien los botones son materiales habituales en los registros arqueológicos históricos, sus bajas cantidades los han vuelto objeto de descripciones generales de su materialidad (madera, hueso, nácar, etc.) dentro de estudios centrados en otras materialidades más ubicuas y cuantitativamente entendidas como significativas (cerámicas, restos arqueofaunísticos, etc.). En el caso del sito La Basurita, los botones en general y particularmente los botones tipo Prosser constituyen un registro cuantitativamente interesante para analizarlos en el contexto de la expansión del capitalismo industrial de mediados del siglo XIX y como una vía de análisis más de la inclusión de la creciente ciudad de Rosario dentro de las lógicas del consumo de este sistema. Otros trabajos han hecho lo propio con las materialidades más habituales dentro de la arqueología histórica, esto es, lozas y restos arqueofaunísticos, como así también metales (Colasuro y Villani, 2006; Raies, 2011a, 2011b y 2013; Colasurdo, 2009, 2011, 2012, 2014a, 2014b, 2014c). No obstante, es interesante considerar a los botones tipo Prosser como un indicador del incremento de prácticas de consumidor -en sentido capitalista- dado su acotada y confiable cronología de inicio y expansión mercantil. Considerado en relación a otros sitios arqueológicos de la misma ciudad de Rosario pero de cronología anterior, es clara la preeminencia de botones de madera/hueso y/o nácar en relación a los Prosser. Así, el sitio JMR, un pozo de basura doméstico localizado en el casco histórico de Rosario, y cuyo uso final se estima hacia principios/mediados de la década de 1850 (Paula Villani, comunican personal, 2016.), cuenta con doce botones, de los cuales ocho son de hueso, uno de metal y tres tipo Prosser (de diferentes subtipos). Los botones representan el 0.5\% del registro de 2620 restos recuperados de JMR (incluyendo materiales óseos, vítreos y cerámicos). Aun cuando los botones Prosser ya estaban en plena producción y expansión comercial, Rosario estaba sólo iniciando su proceso de inclusión dentro del capitalismo industrial, y todavía carecía de una población cuantitativa y cualitativamente consumidora en los términos de este sistema. Sólo en los años siguientes, con la llegada de las masas de migrantes principalmente europeos, es que se acelerarían las prácticas de consumo de mercaderías de todo tipo, incluyendo los botones tipo Prosser, lo que se evidencia en el sitio La Basurita -con un período oficial de uso entre 1873 y 1890. El sitio Arenales (Ar), por su parte, también un pozo de basura doméstico fuera del casco histórico, con una cronología asignada de mediados del siglo XIX (Colasurdo, 2013, 2014b), cuenta con unos 800 restos recuperados de las mismas materialidades, y el único botón presente es de metal. No obstante la cronología antedicha, es posible que la misma sea anterior, dado que las lozas utilizadas para establecer la misma -pintadas a mano y transfer printsean indicativas de un uso más cercano a las primeras décadas del siglo XIX que de mediados del mismo. La total ausencia de botones Prosser se suma a la ausencia de lozas del tipo de la segunda revolución industrial, indicando una cronología anterior a la asignada al sitio, resaltando el interés indicativo de estos botones para la ciudad durante la segunda mitad del siglo XIX.

Es claro que los botones Prosser poseen un gran potencial como indicador cronológico post quem 1840 para la arqueología histórica en general, y en el caso específico de la ciudad de Rosario en el contexto de los sitios arqueológicos de mediados del siglo XIX, es notable el "salto" que se produce en LB de los botones tipo Prosser en relación con las materialidades existentes con anterioridad al proceso industrial Prosser, lo que indica una clara tendencia hacia el reemplazo de botones de nácar, hueso, madera y metal por la nueva tecnología, que además ofrecía una interesante variedad de formas, tipos y colores. Esta predisposición se puede visualizar en el registro arqueológico del sitio JMR, con un final de uso hacia principios/mediados de la década de 1850, y que dentro de una muestra total de doce botones distribuidos en ocho de hueso y uno metálico, registra tres botones Prosser. El sitio Arenales, con una cronología de mediados del siglo XIX (Colasurdo, 2013 y 2014b), está 
siendo reevaluada ya que las lozas presentes son de producción más temprana; Esto también puede significar que sería producto de una cuidadosa estrategia de conservación por parte de los usuarios, por lo tanto es necesario rever esta colección para asignar una cronología indudable. Asimismo la marcada prevalencia de restos de Bos Taurus y Gallusgallus en el registro arqueofaunístico en relación a Ovisaries sería también un indicador de ocupación más temprana. El sitio Casa Parroquial, ubicado en el casco histórico de la ciudad, no es un sitio de descarte en sentido estricto sino un espacio con una variedad de usos (enterratorios humanos, habitaciones, depósito de escombros, por ejemplo) desde principios del siglo XVIII hasta finales del siglo XX El sector del sitio fue utilizado hasta principios del siglo XIX posee un registro material de más de mil restos arqueológicos, entre los cuales sólo hay cuatro botones de vidrio y ningún Prosser. De acuerdo a lo expuesto,existe una marcada presencia de botones Prosser en relación a la disminución consecuente de botones de hueso, madera y nácar, con lo cual se puede asumir, al menos para la ciudad de Rosario, como indicativo de una cronología posterior a 1870, momento en el cual la ciudad se expande demográficamente con la llegada de poblaciones mayormente europeas, y la inserción en la modernidad y el mercado capitalista mundial.

\section{Consideraciones finales}

En conclusión podemos decir que ésta es una interesante vía de análisis que junto con otras materialidades propias del contexto de la arqueología histórica (lozas, gres y restos arqueofaunísticos), se presenta como un buen potencial para ajustar cronologías, en principio para la ciudad de Rosario y también a otros contextos arqueológicos teniendo en cuenta los procesos locales propios en relación a su inclusión al sistema capitalista.

\section{Referencias}

COLASURDO, M. B. (2009). Análisis arqueofaunístico del sitio “Juan Manuel de Rosas” (Rosario, Santa Fe). (Tesis de grado). Escuela de Antropología. Facultad de Humanidades y Artes, UNR, MS.

COLASURDO, M. B. (2011). Análisis arqueofaunístico de un basurero de fines del siglo XIX de la ciudad de Rosario. En: Feuillet Terzaghi, M. R., Colasurdo, M. B., Sartori, J. I., y S. Escudero (Eds) Avances y Perspectivas en la Arqueología del Nordeste. (pp.100-125). Rosario: ST Servicios Gráficos

COLASURDO, M. B. (2012). Análisis del registro arqueológico de dos basureros del siglo XIX de la ciudad de Rosario: primeras aproximaciones. Anuario de Arqueología, 4, 269-281.

COLASURDO, M. B. (2014a). Pautas de consumo de los primeros pobladores de Rosario (Santa Fe, Argentina). Zooarqueología Histórica de un pozo de basura del siglo XVIII. Vestigios, Revista Latino Americana de Arqueología Histórica. Año 8, núm. 2, 7-41.

COLASURDO, M. B. (2014b). Presencia de huellas antrópicas en restos Zooarqueológicos de sitios históricos de los siglos XVIII y XIX en la ciudad de Rosario (Santa Fe, Argentina). Revista de Arqueología Histórica Argentina y Latinoamericana, año 8, núm. 2, 63-82.

COLASURDO, M. B. (2014c). Registro arqueológico y modelos de conducta del consumidor. Cambios en el estilo de vida en Rosario durante los siglos XVIII y XIX. (Tesis Doctoral). Escuela de Graduados, Facultad de Humanidades y Artes, UNR. Argentina. 
COLASURDO, M. B. y VILLANI, M. P. (2006). Aproximaciones metodológicas al análisis de restos arqueofaunísticos y cerámicos del sitio JMR. Actas del XXVI Encuentro de Geohistoria. (pp.93-98). Resistencia: IIGHI. CONICET.

COX, A. (1996). Post-medieval dress accessories from recent urban excavations in Scotland. Tayside and Fife Archaeological Journal, año 2, 52-59.

EBELMEN, J. J. y SALVETAT., L. A. (1855). Recueil des travauxscientifiques, Volume 1. MalletBachelier.

GARCÍA, V. (2006). Una historia transparente. Los vidrios arqueológicos procedentes de las excavaciones en la manzana mercedaria. En Arqueología en el Predio Mercedario de la Ciudad de Mendoza. Mendoza: FFyL, UNC, (eds).

GIOVANETTI, M., N., GONZALES BENEGAS, M. T., IGLESIAS y V. LERMA. (2001). Sitio Iraola. Avance en el tratamiento de los materiales. Actas XIV Congreso Nacional de Arqueología Argentina, Tomo 1. (pp. 579-586). Rosario: FHyA, UNR.

GORSKI, J. (2009). Warman's Buttons Field Guide.Values and indentification. Wisconsin, Estados Unidos: Krause Publications

LANZA, M. M, HERNÁNDEZ DE LARA, O., ALANÍS, S., STORCHI LOBOS, D. y PINOCHET. C. (2015). La batalla de Vuelta de Obligado: primeros resultados de las prospecciones sistemáticas en el área del campamento. Antropología del Museo de Entre Ríos, año 1, núm.1, 86-95.

LAMM, R., LORAH, B., LORAH, L., H. SCHULER, W., SMITH ALBERT, L. y FORD SMITH. J. (1970). Guidelines for Collecting China Buttons. Baubles and Buttons. Guidelines and Resources for Collecting Vintage Buttons 2012. Guidelines for Collecting China Buttons. Recuperado de <http:// baublesandbuttons.com/>

NBS-NATIONAL BUTTON SOCIETY. (2011-2012). Official NBS Classification. National Button Society. Recuperado de <http://www.nationalbuttonsociety.org/>

RAIES, A. (2011a). A La Basurita con los metales. Uso y descarte de artefactos metálicos en Rosario hacia finales del siglo XIX. Una perspectiva arqueológica. (Tesis de grado). Escuela de Antropología. Facultad de Humanidades y Artes, UNR, MS.

RAIES, A. (2011b). Arqueología Urbana de Rosario. Análisis de los precintos de bebidas del sitio La Basurita (1870 -1890). Actas del $1^{\circ}$ Congreso Internacional de Arqueología de la Cuenca del Plata4to. Encuentro de Discusión Arqueológica del Nordeste Argentino- II Jornadas de Actualización en Arqueología Tupiguaraní. Buenos Aires.

RAIES, A. (2013). Arqueología urbana de Rosario. Análisis de los precintos de bebidas del sitio la basurita (1870 -1890). Cuadernos INAPL-Series Especiales-, año 1, núm. 3, 96-104.

RIVERS, S.(1999). An analysis of the buttons from three historic homes in western Kentucky. Ohio Valley Historical Archaeology, núm. 14, 29-35.

SCHÁVELZON, D.1991. Arqueología histórica de Buenos Aires. Buenos Aires. La cultura material porteña de los siglos XVIII y XIX. Buenos Aires: Corregidor.

SPRAGUE, R. (2002). China or Prosser button. Identification and dating. Historical Archaeology, año 36, núm. 2, 111-127. 
VOLPE, S. (1988). Programa de investigación del Área Arqueológica Urbana "Bajo Sur". Museo de la Ciudad. Rosario. MS.

VOLPE, S. (1992). Arqueología Urbana de Rosario, Sitios MCU 1 y MCU 6. Jornadas de historia de Rosario. Rosario. MS.

VOLPE, S. (1994). Catálogo de vajillas de loza Inglesa en Rosario. Municipalidad de Rosario, Rosario: Editorial Municipal.

Recibido: $31 / 03 / 2017$

Aceptado: 27/08/2017 